\title{
Erratum to: development and testing of a mobile application to support diabetes self-management for people with newly diagnosed type 2 diabetes: a design thinking case study
}

Mira Petersen and Nana F. Hempler*

\section{Erratum}

After publication of the original article [1] it was noted that Figs. 1 and 2 had been swapped.

Please note that figure one should be swapped with figure two and the corresponding caption. These errors were introduced during typesetting; thus the publisher apologizes for this error. Below are figures one and two with the correct corresponding captions.

Published online: 12 September 2017

\section{Reference}

1. Petersen M, Hempler NF. Development and testing of a mobile application to support diabetes self-management for people with newly diagnosed type 2 diabetes: a design thinking case study. BMC Med Inform Decis Mak. 2017 Jun 26;17(1):91

* Correspondence: nana.folmann.hempler@regionh.dk

Health Promotion Research, Steno Diabetes Center Copenhagen

Copenhagen, Denmark 
My overview: Personalized overview of diabetes care activities, a calendar of appointments, and roles of the patient

Status: Recording of data related to well-being, sleep, and stress

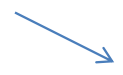

My health data: Recording of health data over time $\longrightarrow$ (e.g., blood sugar, HbA1c, blood pressure, weight, BMI)

Fig. 1 App functions and healthcare professionals

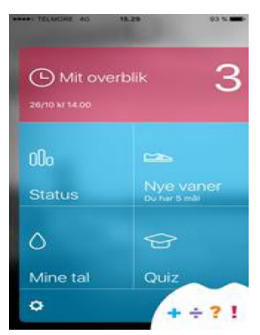

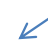

New habits: Reflections about challenges, tests, and goal-setting

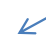

Quiz: Knowledge games related to $\checkmark$ diabetes (e.g., exercise, type 2 diabetes, foot care, diet)

\section{Workshop 1}

Aim: To validate the results

from workshop, observations

and interviews

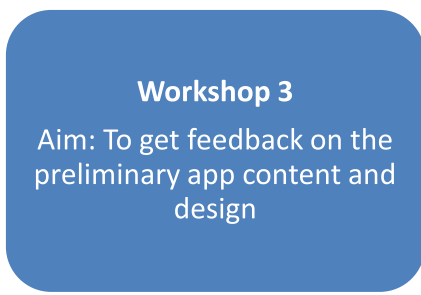

Workshop 5

Aim: To refine and adjust the

wireframes concerning app content and design
- Needs and challenges identified in interviews and observation were recognised by participants.

- Findings include 1) invisible bodily infirmities and no sense of bodily change as a result of diabetes, 2) only few had emotional reactions and worries related to diabetes, 3) few changes in daily routines and lifestyle, 4) challenging relations with healthcare personnel (too little time for dialog).
- Participants preferred most the idea of having a graph showing associations between self-selected parameters of importance to them e.g. sleep, blood glucose, diet, and well-being

- Participants were positive towards ideas of having an overview of services and possibilities available for newly diagnosed with diabetes, integration of own questions as preparation for consultations with physicians, and identification and priotization of needs and challenges related to diabetes.

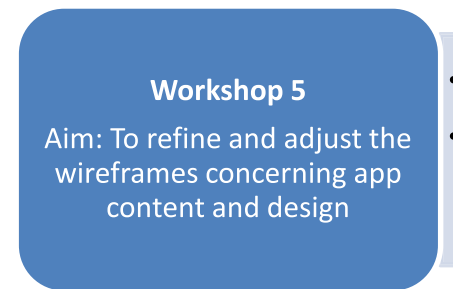

-Discussion of wording in the app eg. 'diabetes stress' or 'stress' and the use of smileys, colors etc.

- Participants requested more concise diabetes-specific information they could easily access when they needed it, such as the difference between blood sugar and glycated hemoglobin (HbA1c).

Fig. 2 Summary of feedback from workshops with users 